\title{
A Kind of Neither Keynesian Nor Neoclassical Model (3): The Decision of Inflation
}

\author{
Ming'an Zhan', Zhan Zhan² \\ ${ }^{1}$ Yunnan University, Kunming, China \\ ${ }^{2}$ Westa College, Southwest University, Chongqing, China \\ Email:1792481782@qq.com
}

How to cite this paper: Zhan, M.A. and Zhan, Z. (2017) A Kind of Neither Keynesian Nor Neoclassical Model (3): The Decision of Inflation. Open Access Library Journal, 4: e3333.

http://dx.doi.org/10.4236/oalib.1103333

Received: December 26, 2016

Accepted: January 14, 2017

Published: January 18, 2017

Copyright (C 2017 by authors and Open Access Library Inc.

This work is licensed under the Creative Commons Attribution International License (CC BY 4.0).

http://creativecommons.org/licenses/by/4.0/

\begin{abstract}
The difficulty of inflation theory is to explain the fluctuations of the price level in short-term. First, we use the relationship between price and money in the traditional quantity equation to derive the inflation equation that can explain changes of the price level in the long-term and short-term. Then, by analyzing the phase diagram of the single variable and the complex variable in the inflation equation, we find that the fundamental reason of the periodic change of the short-term price is the periodic change of the real interest rate. Because fluctuations of the inflation rate and interest rate are the same in phase, so there is no difference in the business cycle. Finally, this paper analyzes the rise and fall of core price under the influence of money and real interest rate respectively. It lays the foundation for further discussion of the relationship between inflation and unemployment in Phillips curve.
\end{abstract}

\section{Subject Areas}

Economics

\section{Keywords}

Inflation, Interest Rate, Quantity Equation, Phase Diagram

\section{Introduction}

Generally, problems of inflation are regarded as the relation between price and currency, and the price and real output. We need to ask the question that whether currency only has impacts on price or on both price and real output? Friedman concluded that: "Inflation is always and everywhere a monetary phenomenon" [1]. However, since the quantity theory of money cannot explain the change pattern of price in short-term, people are willing to believe that there is a 
certain relation between currency and real output based on Phillips curve [2].

Categorized by the transaction motive, speculative motive and precautionary motive in Keynesian theory, the demand for money has different models: Diamond built up the iterative model based on the storage functionality of money [3]; Baumol-Tobin model provided the analysis of speculation demand for money to bonds [4] [5]; McCallum model analyzed how the demand for money restrained by the transaction cost [6]; models of Patinkin, Whalen, Weinrobe, Frenkel and Jovanobic, Blanchard and Fischer analyzed the precautionary or uncertain demand for money from different aspects [7] [8] [9] [10] [11]. Although different models of demand for money have different preconditions, they all lead to the same conclusion: the demand for money is positively related to the real income and negatively related to the interest rate.

However, statistical data since 1970s have shown that there is no stable functional relation between currency and output as well as currency and interest rate [12]. The concept of "demand for money" may be abandoned in the inflation theory, and it will be easier to find the essence of price changes by analyzing the relationship between any amount of money and real output.

\section{Derivation of Inflation Equation}

Since there is no specific currency variable in Cobb-Douglas function, we cannot deduce the relation among the quantity of money, output and the price lever based only on Cobb-Douglas function. The fundemental function of currency is to increase the exchange efficiency between different kinds of wealth, or in other words, to decrease the transaction cost. Assume the nominal output $Y$ as the exchanging object of currency, then we can get the easiest exchange equation of money:

$$
a M=Y
$$

In which, $a$ refers to the exchange coefficient (varies in terms of different currencies, such as U.S. dollars or Japanese yen), $M$ refers to the quantity of currency or the currency stock, $Y$ refers to the nominal output. To express Equation (1) by growth rate, and $a$ is a constant, then:

$$
\frac{\mathrm{d} M}{M}=\frac{\mathrm{d} Y}{Y} \text {, namely } \dot{M}=\dot{Y}
$$

Since there are statistical data on both sides of the equation (take $M_{2}$ as $M$ in the Equation), we can verify the rationality of Equation (2) in reality. As Figure 1 shows, during 1970-2015 statistics in the United States, although the direction of fluctuation of $\dot{Y}$ and $\dot{M}_{2}$ has significant difference, the average of $\dot{Y}=$ 0.0649 , which is quite close to the average of $\dot{M}_{2}=0.0685$, so there is still a link between $\dot{Y}$ and $\dot{M}_{2}$.

We have calculated that using $M_{2}$ as the $M$ is a better to use other monetary indices (such as $M_{0}$ or $M_{1}$ ) in Equation (2). Therefore, according to statistical data in the United States, we can regard $M_{2}$ as $M$ in our theoretical analysis.

The nominal output $Y$ is the multiplier of price index $P$ and the real output $Y_{r}$, namely $Y=P Y_{r}$, then Equation (2) can be expressed as: 


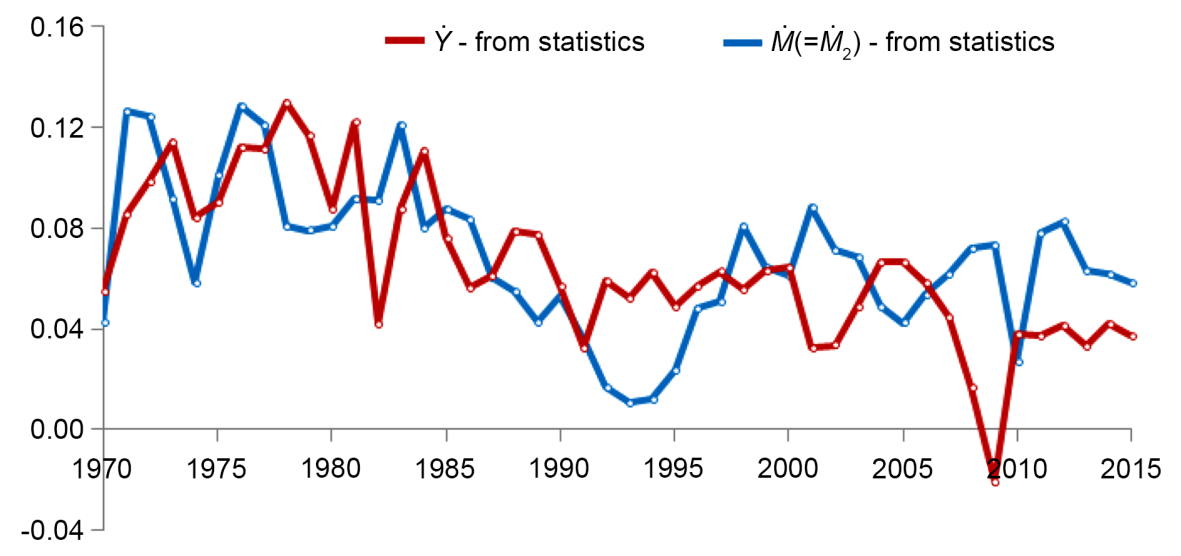

Figure 1. Relations between statistical data of $\dot{Y}$ and $\dot{M}_{2}$. Calculation and resources: $\dot{Y}=\mathrm{d} Y / Y \approx \Delta Y / Y=\Delta \mathrm{GDP} / \mathrm{GDP}, \dot{M}_{2}=\mathrm{d} M_{2} / M_{2} \approx \Delta M_{2} / M_{2}$. Data of GDP are from http://www.bea.gov. Data of $M_{2}$ are from Table-H.6 in http://www.federalreserve.gov.

$$
\frac{\mathrm{d} M}{M}=\frac{\mathrm{d} Y_{r}}{Y_{r}}+\frac{\mathrm{d} P}{P}, \text { namely } \dot{M}=\dot{Y}_{r}+\dot{P}
$$

In the Equation (3), $\dot{M}$ can be observed directly from the statistical data, but only one of $\dot{Y}_{r}$ and $\dot{P}$ is from the statistical data, and the other can be calculated indirectly by the definition $Y=P Y_{r}$. Friedman and Schwartz states in "A Monetary History of the United States, 1867-1960" that there is a fact but not strict relation between the growth rate of currency and price [13]. As shown in Figure 2, there are relations between $\dot{P}$ and $\dot{M}$ in long-term trend, but fluctuation directions are very different in the short-term.

In order to explain the difference of $\dot{P}$ and $\dot{M}$ in the fluctuation, we define the residual of $\dot{M}$ and $\dot{P}$ as $\dot{V}$, namely $\dot{V}=\dot{Y}-\dot{M}$, and observe the direction of fluctuation of $\dot{V}$ and $\dot{M}$. As Figure 3 shows, $\dot{V}$ and $\dot{M}$ change reversely in short-term. Since $\dot{Y}$ includes the change of price index $P$ and that of real output $Y_{r}$, unless we have already known the relation between $P$ and $V$, or $Y_{r}$ and $V$, otherwise it is impossible to explain the reverse changing trend of $\dot{V}$ and $\dot{M}$ in short-term.

In fact, $\dot{V}=\dot{Y}-\dot{M}$ equals to $\mathrm{d}(\ln V)=\mathrm{d}(\ln Y)-\mathrm{d}(\ln M)=\mathrm{d}[\ln (Y / M)]$. By neglecting initial conditions, we can rewrite it as $V=Y / M$. Since $Y=P Y_{r}$, then:

$$
M V=P Y_{r}, \text { or } \dot{M}+\dot{V}=\dot{P}+\dot{Y}_{r}
$$

The above equation is the famous Fisher equation with different symbols from the original equation [14], in which $V$ is called the velocity of currency flow. Although there are more variables in Equation (4) compares to that in Equation (3), it still does not provide more information to analyze the short-term change of $P$, unless we clarify the relation between $V$ and other variables. If we assume $V$ as a constant as Keynes and Friedman did [15] [16], then Equation (4) became Equation (3), so it is unhelpful for us to explain the change of $P$ in short-term.

From the above derivation process, we can see that Equation (4) is not sacred by people's imagination, but is an identity defined by the hypothesis $\dot{V}=\dot{Y}-\dot{M}$. 


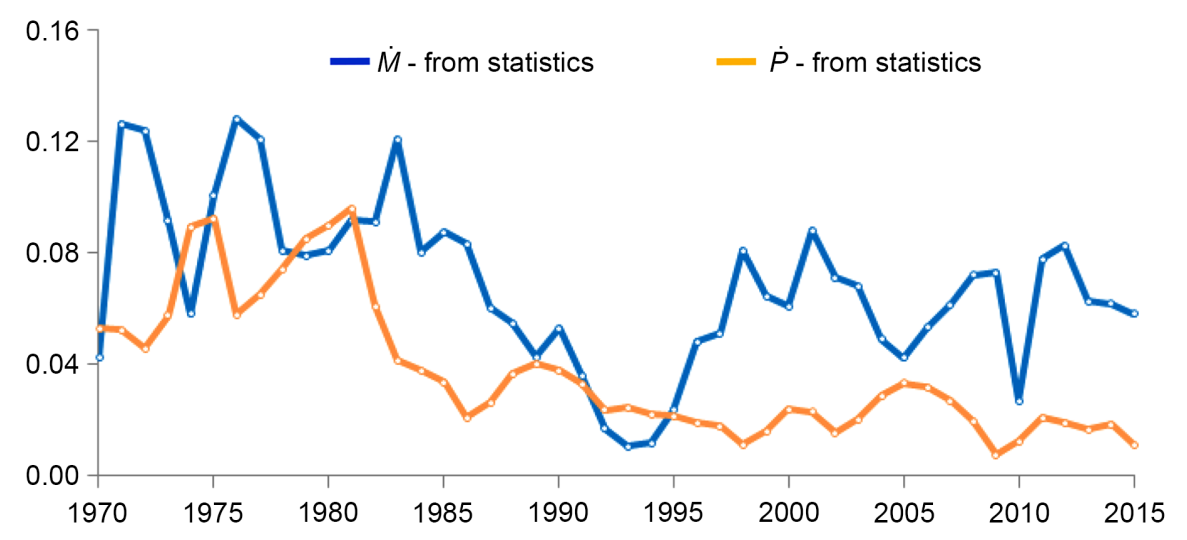

Figure 2. Relations between statistical data of $\dot{P}$ and $\dot{M}\left(\dot{M}_{2}\right)$. Calculation and resources:

1) $\dot{M}=\dot{M}_{2}$, data of $\dot{M}_{2}$ see Figure 1.2) The inflation rate $\dot{P}=\mathrm{d} P / P \approx \Delta P / P$. The price index $P$ is calculated by nominal GDP and real GDP, data of nominal and real GDP are from http://www.bea.gov.

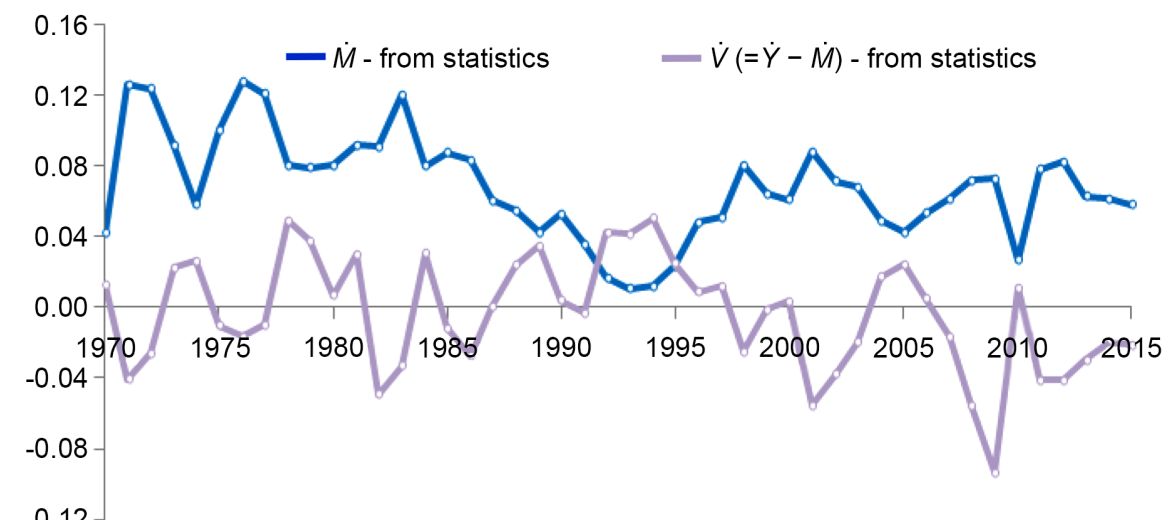

Figure 3. Relations between $\dot{V}$ and $\dot{M}$. Calculation and resources: $\dot{V}=\dot{Y}-\dot{M}$. Data of $\dot{Y}$ and $\dot{M}$ see Figure 1 .

It is easy to fall into the trap of subjective judgment or cyclic interpretation, as the various explanations based on the identity $Y=C+I_{g}+(X-M)$.

In Equation (4), $M$ and $Y_{r}$ are exogenous variables, in which $M$ is generally determined by the monetary authority, and $Y_{r}$ is theoretically determined by Cobb-Douglas function. We cannot rewrite Equation (4) into $Y_{r}=M V / P$ and regard $M$ and $P$ as reasons for the change of $Y_{r}$. Otherwise, when $V$ is a constant, the real output can be increased as long as the money supply is increased, as the IS-LM model does. Unfortunately, in traditional macroeconomics, there are many such subjective theories.

Under the thought of the exchanging proportion of currency and real wealth is varying along with time, then Equation (4) is a quantity equation of currency and real wealth at a certain point of time. At time point $t$, the monetary income collected at $t$ would suffer lose when it is exchanged to real wealth at time point $(t+1)$ due to the increase of price. In that case, the most rational chose in a inflationary environment should be: in consumption area, to exchange monetary income into real wealth at each point of time; in investment area, to switch in- 
vestment objects on those object whose nominal revenue could reflects the latest inflation rate. The assumption above indicates that the change of price would affect the velocity of currency flow. We assume the velocity of currency flow is the function of inflation rate, namely $V=f(\dot{P})$. Since:

$$
\mathrm{d} V=\mathrm{d} f(\dot{P})=\frac{\mathrm{d} f(\dot{P})}{\mathrm{d} \dot{P}} \cdot \frac{\mathrm{d} \dot{P}}{\dot{P}} \cdot \dot{P}, \text { simplified as } \mathrm{d} f(\dot{P})=f^{\prime}(\dot{P}) \ddot{P} \dot{P}
$$

then

$$
\dot{V}=\frac{\mathrm{d} V}{V}=\frac{\mathrm{d} f(\dot{P})}{f(\dot{P})}=\frac{\dot{P} f^{\prime}(\dot{P})}{f(\dot{P})} \cdot \ddot{P}
$$

Substitute Equation (5) into (4),

$$
\dot{M}=-\dot{V}+\dot{P}+\dot{Y}_{r}=-\frac{\dot{P} \ddot{P} f^{\prime}(\dot{P})}{f(\dot{P})}+\dot{P}+\dot{Y}_{r}=\dot{P}\left[1-\frac{\ddot{P} f^{\prime}(\dot{P})}{f(\dot{P})}\right]+\dot{Y}_{r},
$$

therefore

$$
\dot{P}=\frac{\dot{M}-\dot{Y}_{r}}{1-\frac{f^{\prime}(\dot{P})}{f(\dot{P})} \cdot \ddot{P}}
$$

Equation (6) indicates that the change of $\dot{P}$ is not only related to $\left(\dot{M}-\dot{Y}_{r}\right)$, but also to $f(\dot{P})$ and the growth rate of $\dot{P}$ in short-term. In long-term, the average value of $f^{\prime}(\dot{P})$ and $\ddot{P}$ are equal to 0 , otherwise there is a contradiction between Equation (6) and $\dot{P}=\dot{M}-\dot{Y}_{r}$ in Equation (3). In that case, we need to verify if the average value of $\ddot{P}$ or $f^{\prime}(\dot{P})$ is equal to 0 .

Since we do not know the details of the function $V=f(\dot{P})$, it is impossible to verify the average value of $f^{\prime}(\dot{P})$. However, we could estimate the average value of $\ddot{P}$ by observing statistical data of $\dot{P}$. As Figure 4 shows, during 1970-2015, the trend line of $\ddot{P}$ is horizontal and it's the average value in longterm is 0.01 , which approximately equal to 0 .

If the average value of $\dot{M}$ and $\dot{Y}$ in long-term are the same, then their residual $\dot{V}$ should be equal to 0 . In short-term, when the direction of fluctuation of $\dot{V}$ and $\ddot{P}$ are the same, then we can assume the changing pattern of $\dot{V}$ is determined by $\ddot{P}$. Make $\theta=\dot{P} f^{\prime}(\dot{P}) / f(\dot{P})$, and according to Equation (5), then $\dot{V}=\theta \ddot{P}$. In Figure 4, we can see that the long-term trend of $\dot{V}$ and $\ddot{P}$ is consistent (their trend lines are parallel), and the short-term fluctuation direction of $\ddot{P}$ is also related. If the range of fluctuation of $\theta$ is significantly narrower than that of $\ddot{P}$ in $\dot{V}=\theta \ddot{P}$, then we can take $\theta$ as a constant.

In fact, the value of $\theta$ is not important in the long-term, because as long as the long-term average of $\ddot{P}$ is 0 , there is $\dot{P}=\dot{M}-\dot{Y}_{r}$ according to Equation (6). This is the reason that money is always neutral in long-term, but is not clear in the medium and short term.

As Figure 4 shows, based on statistical data during 1970-2015, make $\theta=0.1$, then the average of $0.1 \ddot{P}$ equals to 0.0010 and its long-term trend is approximately horizontal. The average of $\dot{V}$ equals to -0.0040 , therefore the average 


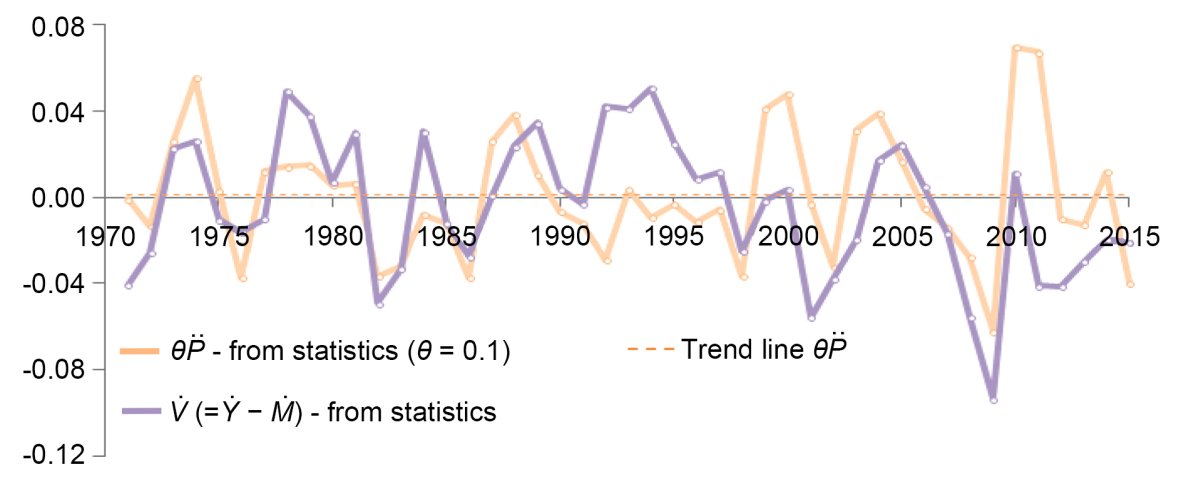

Figure 4. Relations between $\ddot{P}$ and $\dot{V}$. Calculation and resources: 1) $\ddot{P}=\mathrm{d} \dot{P} / \dot{P} \approx \Delta \dot{P} / \dot{P}$, $\dot{P}=\mathrm{d} P / P \approx \Delta P / P$. Data of $P$ see Figure 2. 2) Theoretically, trend of $\theta \ddot{P}$ and $\dot{V}$ are consistent in long-term, and the direction of fluctuation and range of fluctuation should be the same. The difference showed in Figure 4 indicates: $\theta$ is not necessarily a constant. However, it is often regarded as a constant when $\dot{P}$ changes in a certain rand and the change of $\theta$ is comparatively small compared to that of $\ddot{P}$.

of $0.1 \ddot{P}$ is close to that of $\dot{V}$, and the direction of fluctuation of $0.1 \ddot{P}$ is consistent with that of $\dot{V}$, but some differences in the range of fluctuation. Substitute $\dot{V}=\theta \ddot{P}$ into Equation (4), then:

$$
\dot{P}-\theta \ddot{P}=\dot{M}-\dot{Y}_{r}
$$

Equation (7) indicates that the inflation rate $\dot{P}$ is not only affected by exogenous variables $\dot{M}$ and $\dot{Y}_{r}$, but also its own change rate $\ddot{P}$. More importantly, the variables in Equation (7) have corresponding statistical data in the United States, so that we can verify the results of the inference. Since the relationship between $\dot{P}$ and $\ddot{P}$ can be drawn as a phase diagram, therefore the change of difference between $\dot{M}$ and $\dot{Y}_{r}$ determines the change of phase diagram $\dot{P} \sim \ddot{P}$.

\section{Phase Diagram Analysis of Inflation Equation}

In our paper "A Kind of neither Keynesian nor Neoclassical Model (2): The Business Cycle" [17], we use the phase diagram to analyze the periodic fluctuations of $r$ and $\dot{Y}$, and according to changes of phase diagram $r \sim \dot{r}$, we divide the 1970-2015 period into 9 cycles, as shown in Figure 5.

When we analyze the relationship between the variable $P$ and other variables, the other variables should be real variables that are not affected by the price, so that there is no suspicion that the loop is interpreted. $\dot{P}$ is not only related to $\dot{M}$ but also to $\dot{Y}_{r}$ in Equation (7). Theoretically, the periodicity of $\dot{Y}_{r}$ is determined by the periodicity of $r_{r}$, so the fluctuation of $\dot{P}$ is likely to be related to $r_{r}$ or $r$. In Figure 5, $\dot{P}, r$ and $r_{r}$ vary in the time path of the wave patterns, but they are similar on the characteristics in the cycle. According to the Equation (7), we can analyze the relationship between $r_{r}$ and $\dot{P}$ theoretically.

According to time path of $\dot{P}$ in Figure 5, we estimate there might be a phase diagram of $\dot{P}$ and $\theta \ddot{P}$ with circulating characteristics. It can be testified with statistical data, as shown in Figure 6. 


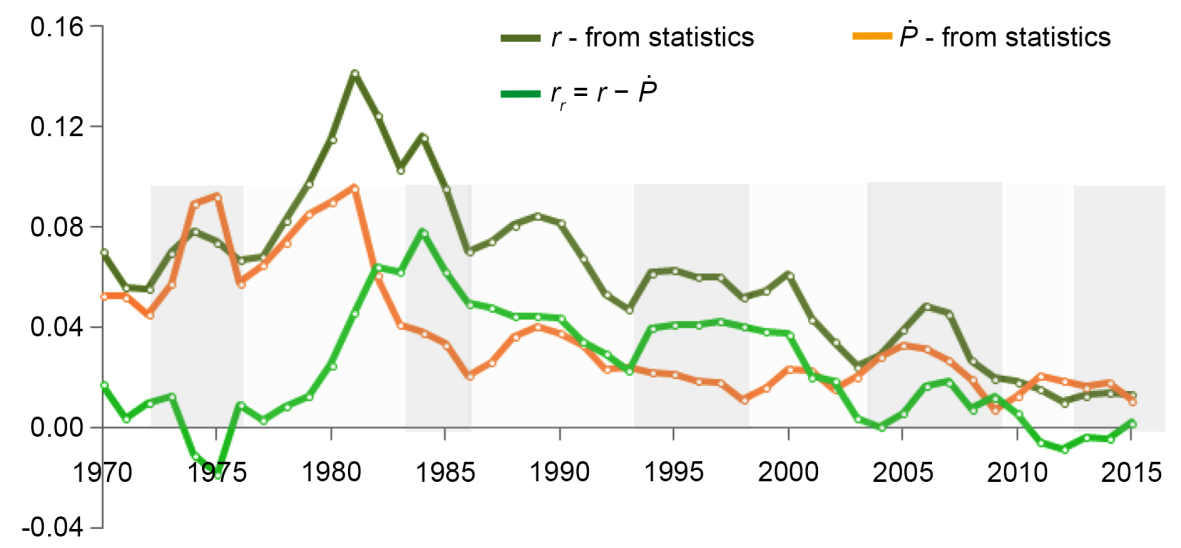

Figure 5. Time paths of $r, r_{r}$ and $\dot{P}$. Calculation and resources: Data of $\dot{P}$ see Figure 2. $r_{r}=r-\dot{P}, r$ is the arithmetic mean of annualized earnings of various US Treasury bonds, data are from http://www.federalreserve.gov/. See paper "A Kind of neither Keynesian nor Neoclassical Model (2): the Business Cycle” [17].

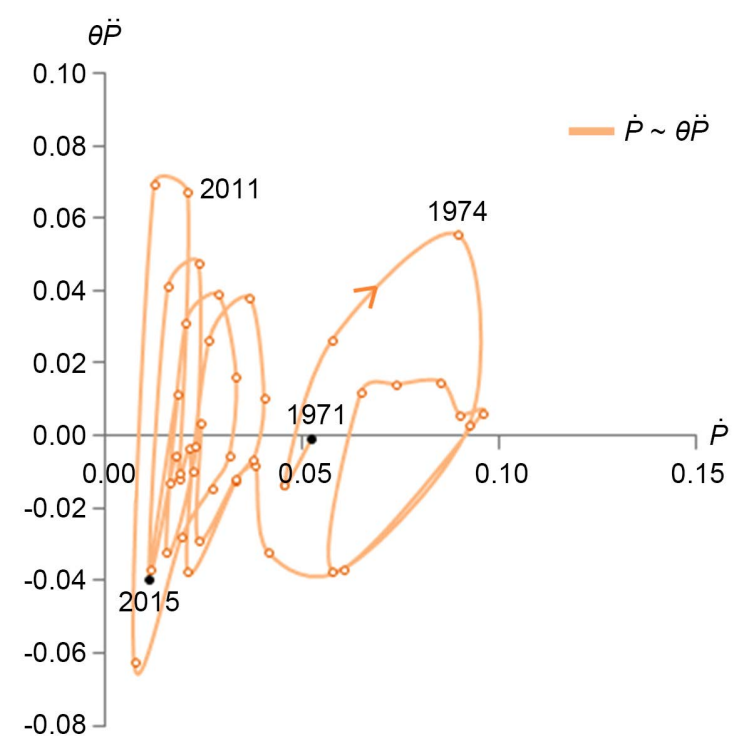

Figure 6. Phase diagram $\dot{P} \sim \theta \ddot{P}$ of statistical data. Calculation and resources: $\theta=0.1$. When $\theta \ddot{P}>0$, then $\dot{P}$ moves to the right; and when $\theta \ddot{P}<0, \dot{P}$ moves to the left. Therefore the phase diagram $\dot{P} \sim \ddot{P}$ rotates clockwise. $\ddot{P}=\mathrm{d} \dot{P} / \dot{P} \approx \Delta \dot{P} / \dot{P}, \dot{P}=\mathrm{d} P / P \approx$ $\triangle P / P$, data of $\dot{P}$ see Figure 2.

The theoretical analysis of the phase diagram $\dot{P} \sim \theta \ddot{P}$ can be seen: the rotation of $\dot{P} \sim \theta \ddot{P}$ must be clockwise based on mathematical logic as shown in Figure 7 (a). Since $\theta \ddot{P}>0$ above the horizontal axis, then $\dot{P}$ should more towards right, vice versa. We call $\overline{\dot{P}}$ in the ellipse as the core inflation rate, which is determine by the average of $\dot{P}$ in a business cycle.

Take $(\dot{P}-\theta \ddot{P})$ in Equation (7) as a composite variable, then we can draw the phase diagram of $\dot{P} \sim(\dot{P}-\theta \ddot{P})$ which rotates counterclockwise as Figure $7(\mathrm{~b})$ shows. The dotted line $\dot{P}=\theta \ddot{P}$ is an auxiliary line for drawing the phase diagram $\dot{P} \sim(\dot{P}-\theta \ddot{P})$. The phase diagram $\dot{P} \sim(\dot{P}-\theta \ddot{P})$ under the bisector $\dot{P}=\theta \ddot{P}$ correspond to parts of $\dot{P} \sim \theta \ddot{P}$ above the horizontal axis, vice versa. 


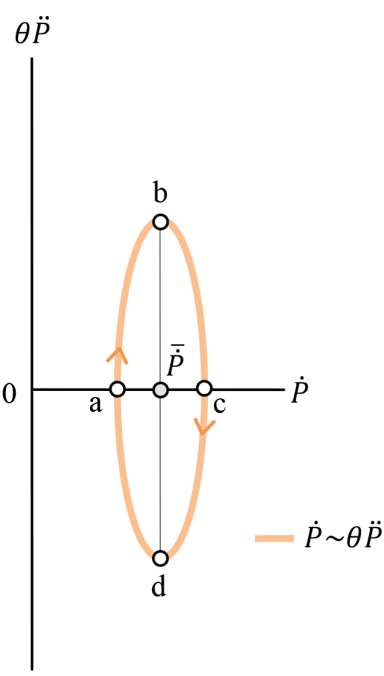

(a)

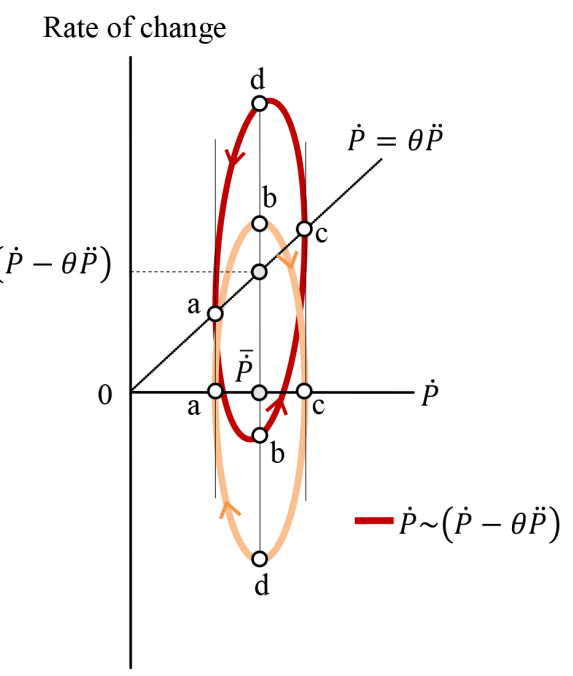

(b)

Figure 7. (a) Standard shape of the phase diagram $\dot{P} \sim \theta \ddot{P}$, (b) Phase diagram $\dot{P} \sim(\dot{P}-\theta \ddot{P})$. Notice: In (a), we firstly draw the auxiliary line $\dot{P}=\theta \ddot{P}$. Point a and $\mathrm{c}$ in the phase diagram $\dot{P} \sim \theta \ddot{P}$ correspond to point a and $\mathrm{c}$ in the phase diagram $\dot{P} \sim(\dot{P}-\theta \ddot{P})$ since $\theta \ddot{P}=0$. Then we can draw other points on the phase diagram based on the composite variable $(\dot{P}-\theta \ddot{P})$. The phase diagram $\dot{P} \sim(\dot{P}-\theta \ddot{P})$ rotates counterclockwise because there is a negative sign in front of $\theta \ddot{P}$.

Since the direction of rotation of $\dot{P} \sim \theta \ddot{P}$ and $\dot{P} \sim(\dot{P}-\theta \ddot{P})$ are reverse, $\dot{P} \sim \theta \ddot{P}$ and $\dot{P} \sim(\dot{P}-\theta \ddot{P})$ will be significant differences in the time path. In Figure $7(\mathrm{~b})$, point $\mathrm{c}$ on the phase diagram $\dot{P} \sim \theta \ddot{P}$ express the maximum value of $\dot{P}$, which locates in the middle of a cycle; point $\mathrm{d}$ on the phase diagram $\dot{P} \sim(\dot{P}-\theta \ddot{P})$ express the maximum value of the composite variable $(\dot{P}-\theta \ddot{P})$, which locates in the second half of a cycle since Point $d$ exists later than point $c$ on the phase diagram $\dot{P} \sim(\dot{P}-\theta \ddot{P})$. As a result, peaks of the composite variable $(\dot{P}-\theta \ddot{P})$ exist later than that of $\dot{P}$ in terms of time paths. This can be verified by statistical data, as shown in Figure 8 .

The average of $\dot{P}$ and the composite variables $(\dot{P}-\theta \ddot{P})$ are 0.035 and 0.036 for 1970-2015 respectively, and their trends are also consistent in longterm. As the Figure 8 shows, $(\dot{P}-\theta \ddot{P})$ have a particularly large peaks and valleys in each cycle. This helps us to confirm the cycle more clearly. Even we can use the phase diagram $\dot{P} \sim \theta \ddot{P}$ or time path of $(\dot{P}-\theta \ddot{P})$ to divide the business cycle in the absence of statistical data $r$.

In the equation $\dot{P}-\theta \ddot{P}=\dot{M}-\dot{Y}_{r}$, since the composite variable $(\dot{P}-\theta \ddot{P})$ on the left side of the equation changes periodically, the composite variable $\left(\dot{M}-\dot{Y}_{r}\right)$ on the right side should also change periodically.

Since the money has a capital attribute, it is assumed that the periodicity of $\dot{M}$ in the short-term may be similar to the periodicity of the capital change $\dot{K}$ in Cobb-Douglas function. By the marginal condition of $Y=A K^{\alpha} L^{\beta}$, $M P K=\partial Y / \partial K=r$, there is $K=\alpha Y / r$, so 


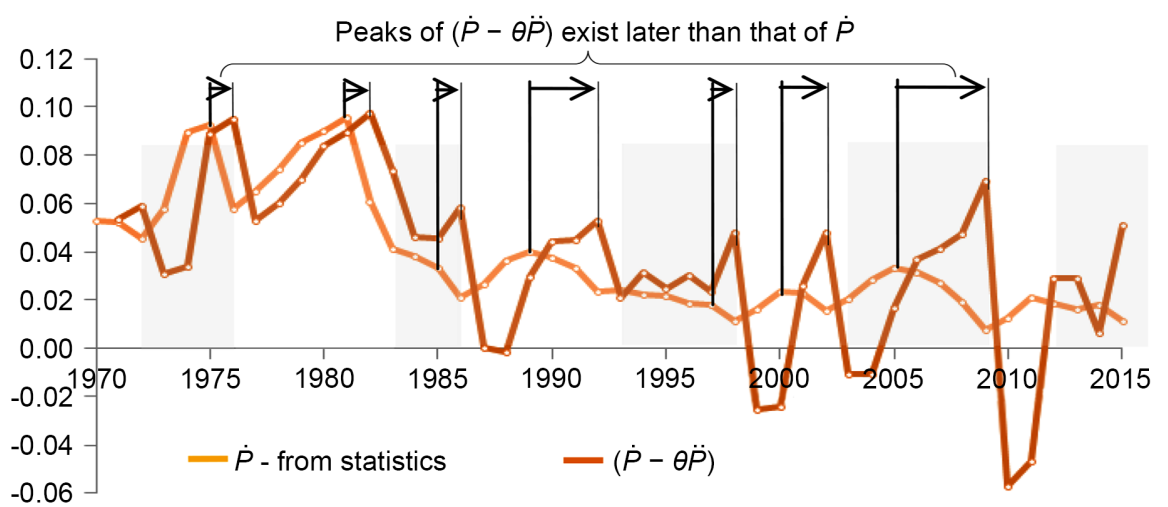

Figure 8. Time paths of $\dot{P}$ and $(\dot{P}-\theta \ddot{P})$. Calculation and resources: $\ddot{P}=\mathrm{d} \dot{P} / \dot{P} \approx$ $\Delta \dot{P} / \dot{P}, \quad \dot{P}=\mathrm{d} P / P \approx \Delta P / P$, data of $P$ see Figure 2. Since $\ddot{P}$ is to be calculated in increments of $\dot{P}$, the initial statistical data of $\ddot{P}$ is one year later than $\dot{P}$. It is 1971 .

$$
\frac{\mathrm{d} K}{K}=\frac{\mathrm{d} Y}{Y}-\frac{\mathrm{d} r}{r}+\frac{\mathrm{d} \alpha}{\alpha},
$$

According to the fundamental equation $\Delta Y=r Y \quad[18]$,

$$
\frac{\mathrm{d} Y}{Y}=\lim _{\Delta Y \rightarrow 0} \frac{\Delta Y}{Y}=\lim _{\Delta Y \rightarrow 0} \frac{r Y}{Y}=r,
$$

therefore, $\mathrm{d} K / K=r-\mathrm{d} r / r+\mathrm{d} \alpha / \alpha$. hypothesis $\mathrm{d} \alpha / \alpha=0$, then

$$
\dot{K}=\frac{\mathrm{d} K}{K}=r-\frac{\mathrm{d} r}{r}=r-\dot{r} .
$$

Compared with $\dot{Y}=r+\dot{r}$, the front of $\dot{r}$ is a negative sign in $\dot{K}=r-\dot{r}$, so the rotation of the phase diagram $r \sim \dot{K}$ is counterclockwise. This causes the peak of $\dot{K}$ to fall behind the peak of $r$ in the time path, as shown in Figure 9. If fluctuation of $\dot{M}$ is similar to $\dot{K}$ in short-term, then the peak of $\dot{M}$ will be in the second half of a cycle, since the peak of $\dot{Y}$ is before the peak of $r$ (the peak of $\dot{Y}$ is in the first half of a cycle).

As guess from Figure 9, if the peak of $\dot{Y}$ is in the first half of a cycle, the peak of $\dot{M}$ which is similar to $\dot{K}$, will be in the second half of a cycle, in other words the peak of $\dot{M}$ will follow the peak of $\dot{Y}$. The statistical data of Figure 10 can verify this conjecture.

Because the phase diagram $r_{r} \sim \dot{M}$ rotates counterclockwise, we can overlap it on the phase diagram $r_{r} \sim\left(-\dot{Y}_{r}\right)$ and then get the phase diagram of the composite variable $\left(\dot{M}-\dot{Y}_{r}\right)$. In this way, the phase diagram $\dot{P} \sim(\dot{P}-\theta \ddot{P})$ and $r_{r} \sim\left(\dot{M}-\dot{Y}_{r}\right)$ can be related according to Equation (7).

Figure 11(a) shows the process to draw the phase diagram $r_{r} \sim\left(\dot{M}-\dot{Y}_{r}\right)$. Firstly we draw the phase diagram $r_{r} \sim \dot{r}_{r}$ rotates clockwise, and draw the phase diagram $r_{r} \sim \dot{Y}_{r}$ rotates clockwise based on equation $\dot{Y}_{r} \approx r_{r}+\dot{r}_{r}$ (The reason for using the symbol $\approx$ will be explained later in the analysis of the Phillips curve). Then we can get the phase diagram $r_{r} \sim\left(-\dot{Y}_{r}\right)$ which is the mirror image of $r_{r} \sim \dot{Y}_{r}$ and rotates counterclockwise.

The phase diagram $r_{r} \sim \dot{M}$ rotates counterclockwise, and the core variable $\overline{\dot{M}}$ is affected by monetary policies and multiple times of the money supply of 


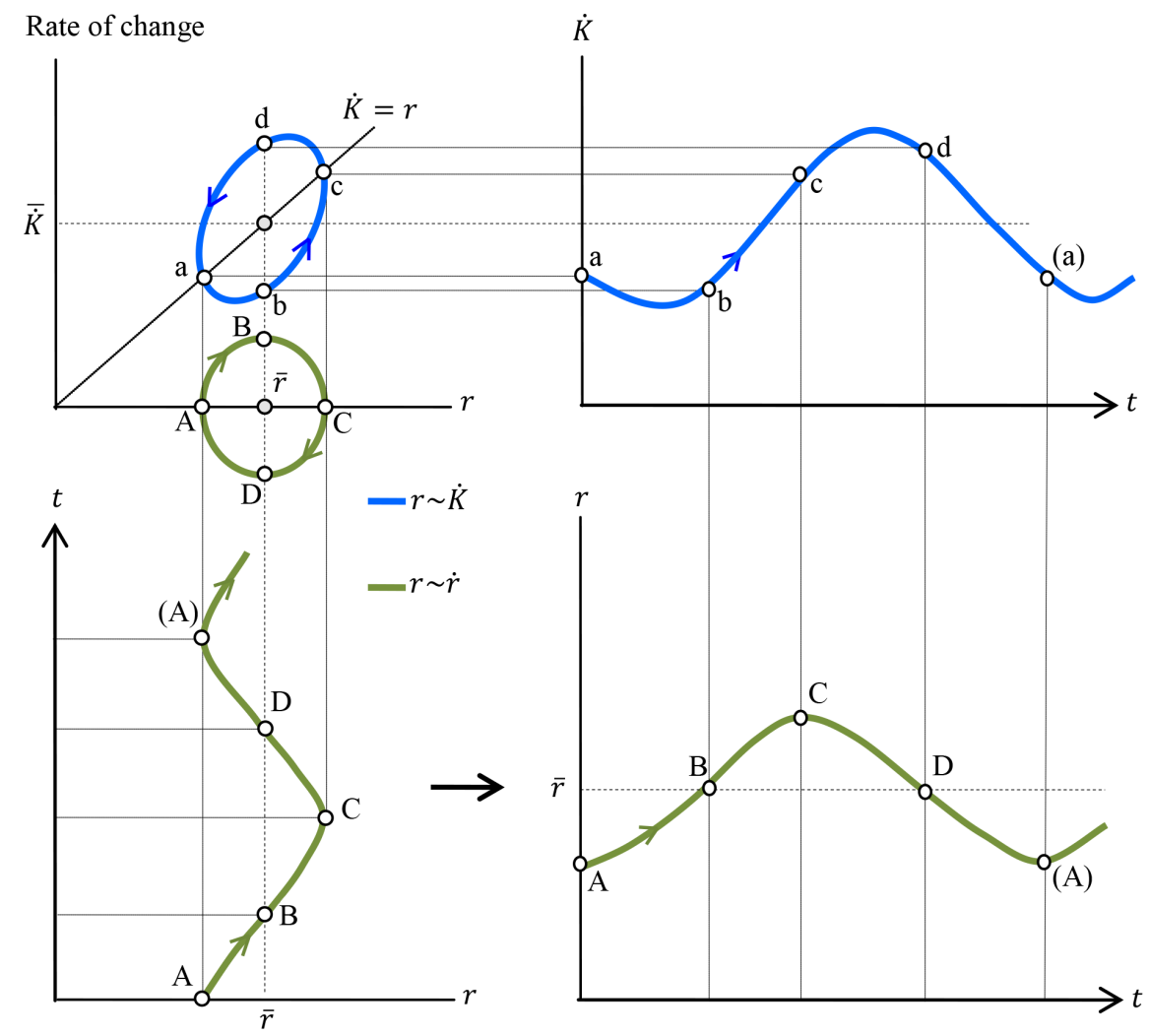

Figure 9. Phase diagram and time path of $\dot{K}$ and $r$. Drawing method see paper "A Kind of neither Keynesian nor Neoclassical Model (2): the Business Cycle” [17].

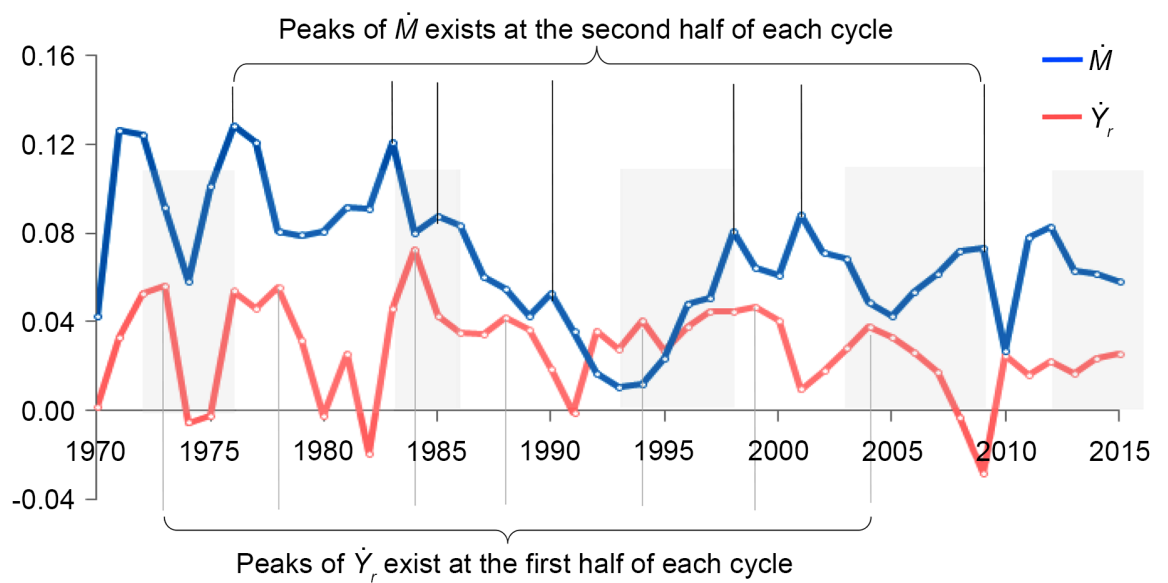

Figure 10. Time paths of $\dot{M}$ and $\dot{Y}_{r}$. Resources: $\dot{Y}_{r}=\dot{Y}-\dot{P}$. Data of $\dot{Y}$ see Figure 1, data of $\dot{P}$ and $\dot{M}$ see Figure 2 .

central bank. The central bank supplies basic currency, and $\overline{\dot{M}}$ comprises of basic currency and other derived currency created by business banks and financial market. The bigger of the monetary multiplier of the basic currency and the derived currency, the higher place on the vertical axis of $\overline{\dot{M}}$. In order to facilitate our narration, we assume $\overline{\dot{M}}$ is determined by the supply of basic money under a constant monetary multiplier even though the multiplier might not be a constant in reality. 
Since the phase diagram $r_{r} \sim \dot{M}$ and $r_{r} \sim\left(-\dot{Y}_{r}\right)$ both involve $r_{r}$, we can overlap corresponding points (point $\mathrm{a}, \mathrm{b}, \mathrm{c}, \mathrm{d}$ ) in these two diagrams and then determine the location, shape and direction of rotation of the phase diagram $r_{r} \sim\left(\dot{M}-\dot{Y}_{r}\right)$ as show in Figure 11(a).

We now could relate the phase diagram $\dot{P} \sim(\dot{P}-\theta \ddot{P})$ to $r_{r} \sim\left(\dot{M}-\dot{Y}_{r}\right)$. Since $\dot{P}-\theta \ddot{P}=\dot{M}-\dot{Y}_{r}$, then the direction of rotation of $r_{r} \sim\left(\dot{M}-\dot{Y}_{r}\right)$ and $\dot{P} \sim(\dot{P}-\theta \ddot{P})$ are the same, and points on $r_{r} \sim\left(\dot{M}-\dot{Y}_{r}\right) \quad$ (as a, b, c, d) are corresponding to that on $\dot{P} \sim(\dot{P}-\theta \ddot{P})$. Since both phase diagrams rotate counterclockwise, then the maximum value of $\left(\dot{M}-\dot{Y}_{r}\right)$ and $(\dot{P}-\theta \ddot{P})$ locates at the second half of a cycle. We can verify this conclusion in statistical data in Figure 12.

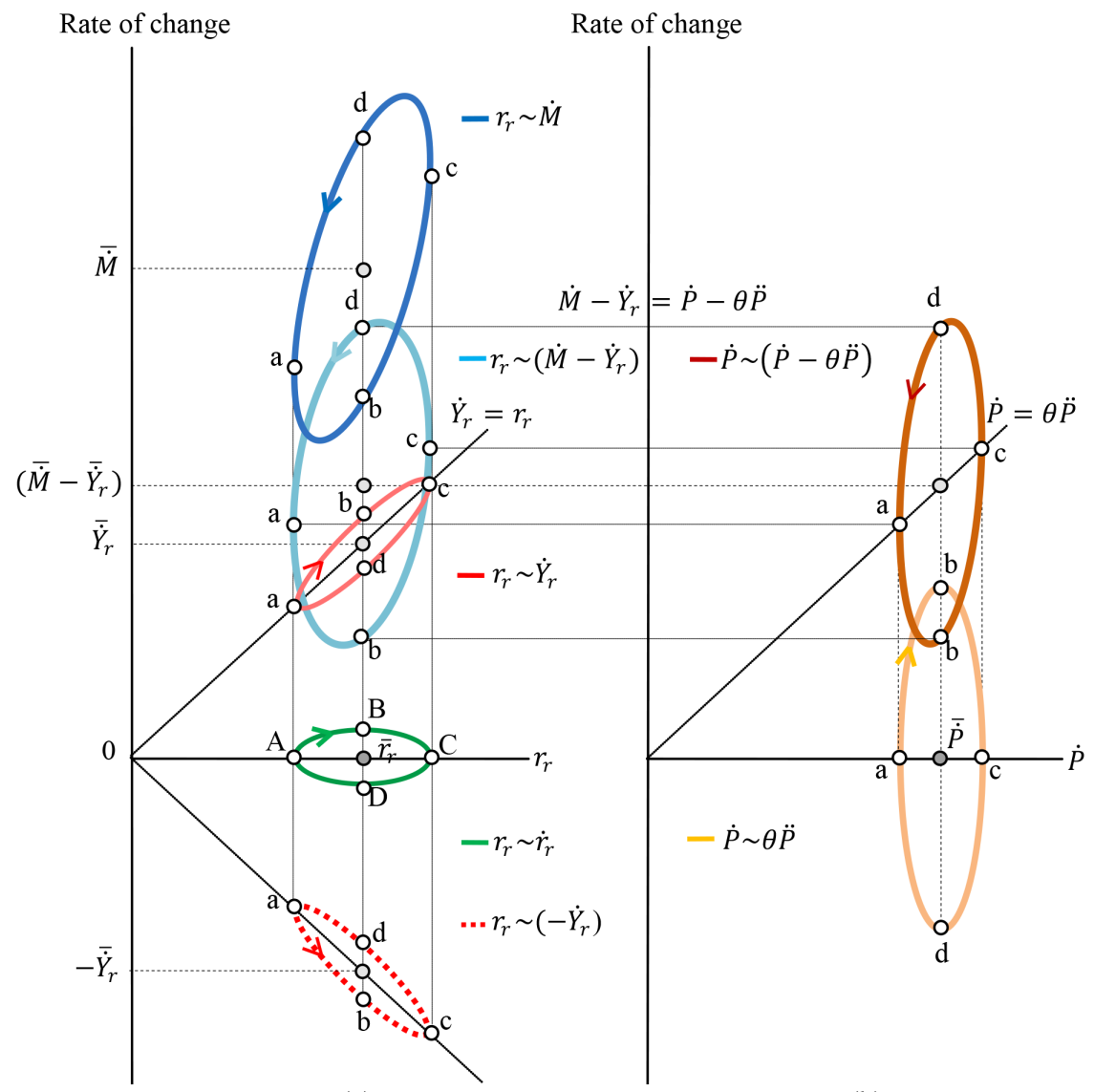

(a)

(b)

Figure 11. (a) Phase diagram $r_{r} \sim \dot{r}_{r}$, (b) Phase diagram $\dot{P} \sim(\dot{P}-\theta \ddot{P})$. Notice: 1) In (a), assume a phase diagram $r_{r} \sim \dot{r}_{r}$ with the core variable $\bar{r}_{r}$. Then draw the phase diagram $r_{r} \sim \dot{Y}_{r}$ with the core variable $\bar{Y}_{r}, \dot{Y}_{r}=r_{r}+\dot{r}_{r}$, therefore $\bar{Y}_{r}=\bar{r}_{r}$. The phase diagram $r_{r} \sim\left(-\dot{Y}_{r}\right)$ is the mirror image of phase diagram $r_{r} \sim \dot{Y}_{r}$. We can get the phase diagram $r_{r} \sim\left(\dot{M}-\dot{Y}_{r}\right)$ by overlapping $r_{r} \sim \dot{M}$ and $\left.r_{r} \sim\left(-\dot{Y}_{r}\right) .2\right)$ (b), firstly draw the phase diagram $\dot{P} \sim \theta \ddot{P}$, then the corresponding diagram $\dot{P} \sim(\dot{P}-\theta \ddot{P})$. Then adjust the location and shape of the phase diagram $\dot{P} \sim \theta \ddot{P}$ based on $r_{r} \sim\left(\dot{M}-\dot{Y}_{r}\right)$ until the phase $\dot{P} \sim(\dot{P}-\theta \ddot{P})$ is corresponding to $r_{r} \sim\left(\dot{M}-\dot{Y}_{r}\right)$, namely $\dot{M}-\dot{Y}_{r}=\dot{P}-\theta \ddot{P}$. 


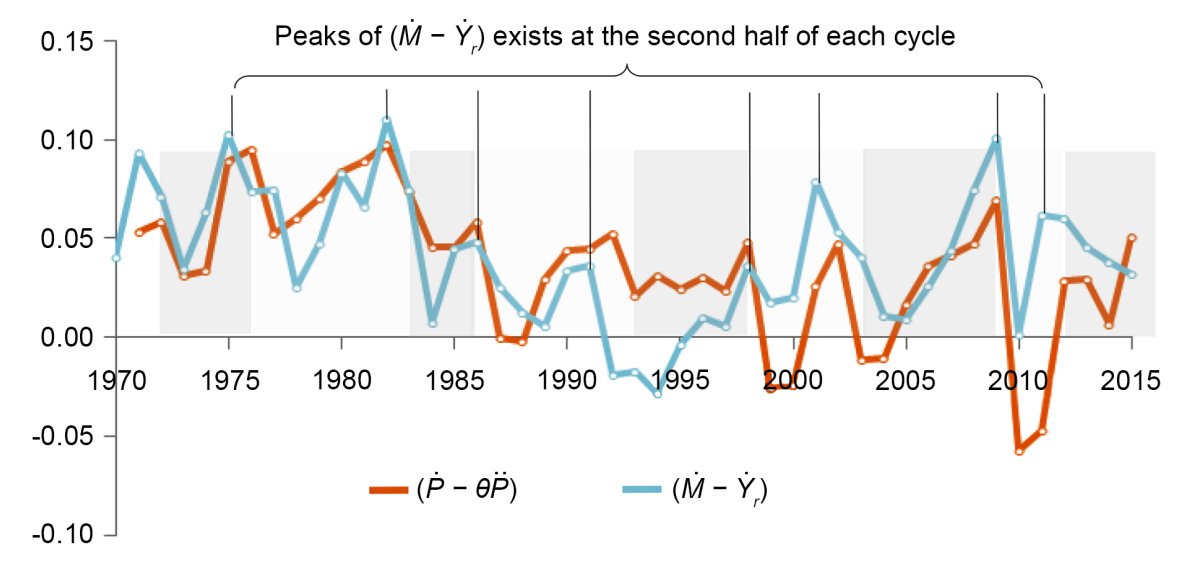

Figure 12. Time paths of $\left(\dot{M}-\dot{Y}_{r}\right)$ and $(\dot{P}-\theta \ddot{P})$. Calculation and resources: $\dot{Y}_{r}=\dot{Y}-\dot{P}$. Data of $\dot{Y}$ see Figure 1, data of $(\dot{P}-\theta \ddot{P})$ see Figure 8 .

In addition, the cycle of $(\dot{P}-\theta \ddot{P})$ and $\left(\dot{M}-\dot{Y}_{r}\right)$ fluctuates in accordance with the inflation equation, but also their long-term average should be equal. The statistical data show that the average value of $(\dot{P}-\theta \ddot{P})$ and $\left(\dot{M}-\dot{Y}_{r}\right)$ are 0.036 and 0.041 for the period 1970-2015. There are two reasons why the average value of $(\dot{P}-\theta \ddot{P})$ is slightly smaller than $\left(\dot{M}-\dot{Y}_{r}\right)$. First, the time of the statistical data is not long enough. The impact of money will lag behind for some time to be reflected in $\dot{P}$, especially in the monetary policy of QE (quantitative easing) over the years. Second, there are leakages of the money, such as part of the currency flow to other countries and regions.

\section{The Change of Price by the Influence of $\overline{\dot{M}}$ and $\overline{r_{r}}$}

Theoretical analysis in Figure 11 show that there are four factors affect the change of macroeconomic price: firstly, the change of $\overline{\dot{M}}$ which is closely related to base money supply; secondly, the periodic change of variable $\tilde{\dot{M}}$; thirdly, the change of core variable $\bar{r}_{r}$ which reflects the operating efficiency of the system; fourthly, the periodic change of $\tilde{r}_{r}$.

In order to identify all above factors, we call $\overline{\dot{M}}, \bar{r}_{r}$ and $\overline{\dot{P}}$ as "core variables" which stand for changes of $\dot{M}, r_{r}$ and $\dot{P}$ due to the migration of phase diagrams $r_{r} \sim \dot{M}, r_{r} \sim \dot{r}_{r}$ and $\dot{P} \sim \theta \ddot{P} . \tilde{\dot{M}}, \tilde{r}_{r}$ and $\tilde{\dot{P}}$ as "fluctuating variables" which stand for changes of $\dot{M}, r_{r}$ and $\dot{P}$ due to the fluctuation of phase diagrams. There relations are: $\dot{M}=\overline{\dot{M}}+\tilde{\dot{M}}, r_{r}=\bar{r}_{r}+\tilde{r}_{r}, \quad \dot{P}=\overline{\dot{P}}+\tilde{\dot{P}}$.

In this way, we can more clearly understand the factors that affect price changes, and get some preliminary inference. For example, with an established phase diagram $r_{r} \sim \dot{r}_{r}$, we can analyze the impact of change of exogenous variable $\overline{\dot{M}}$ on $\dot{P}$.

As Figure 13 shows, assume the initial state of $\overline{\dot{M}}$ as $(\overline{\dot{M}})_{0}$, then we can locate the phase diagram $r_{r} \sim\left(\dot{M}-\dot{Y}_{r}\right)_{0}, \dot{P} \sim(\dot{P}-\theta \ddot{P})_{0}$ and $\dot{P} \sim(\theta \ddot{P})_{0}$ according to $r_{r} \sim \dot{r}_{r}$. Under the effect of monetary policies, $(\overline{\dot{M}})_{0}$ drop to $(\overline{\dot{M}})_{1}$, 

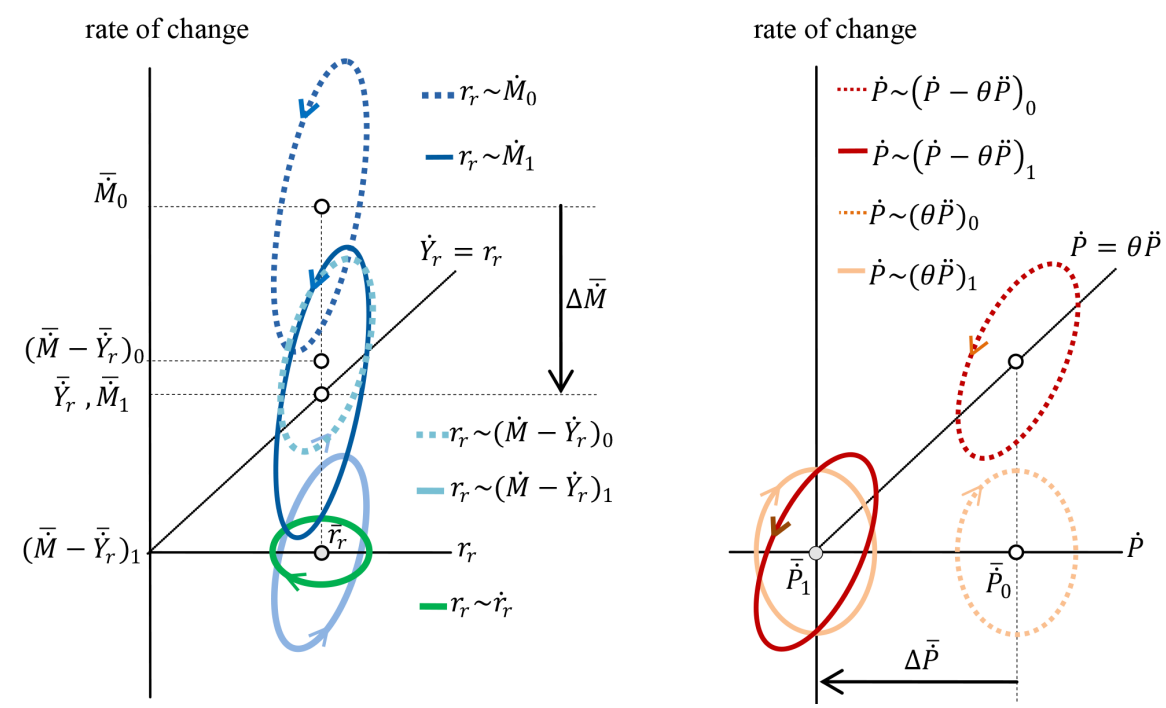

Figure 13. Impacts of changing $\overline{\dot{M}}$ on $\dot{P}$. Notice: 1) This figure is a simplified version of Figure 11, there is no phase diagrams $r_{r} \sim \dot{Y}_{r}$ and $\left.r_{r} \sim\left(-\dot{Y}_{r}\right) .2\right)$ Assume $\bar{r}_{r}$ stay unchanged, then the smaller value of $\overline{\dot{M}}$, the further place of the core variable $\overline{\dot{P}}$ towards left.

and make $(\overline{\dot{M}})_{1}=\overline{\dot{Y}}_{r}$, then $\left(\overline{\dot{M}}-\overline{\dot{Y}}_{r}\right)_{1}$ equals to 0 , therefore $(\overline{\dot{P}}-\theta \overline{\ddot{P}})_{1}=0$, $\overline{\dot{P}}_{1}=0$.

It not only indicates $\overline{\dot{P}}$ and $\overline{\dot{M}}$ change to the same direction, but also the central bank could make the core inflation rate $\bar{P}=0$ by controlling $\overline{\dot{M}}$ (notice: $\bar{P}=0$ is different from $\dot{P}=0$ ). Since $\dot{P}=\dot{P}+\tilde{\dot{P}}$, and $\tilde{\dot{P}}$ fluctuates around $\overline{\dot{P}}$, the value of $\dot{P}$ might be less or more than 0 .

Even though we know that there is relation between the phase diagram $r_{r} \sim \dot{r}_{r}$ and $\dot{P} \sim \theta \ddot{P}$ in Figure 11, we do not know whether the periodic change of $\dot{P}$ is determined by that of $r_{r}$ or the periodic change of $r_{r}$ is determined by that of $\dot{P}$. Since the implicated premise of existence of phase diagrams $r_{r} \sim \dot{M}$ and $\dot{P} \sim \theta \ddot{P}$ is that "money is the trading medium of real wealth", the initial fluctuation is the fluctuation of real interest rate $r_{r}$ and thereafter the fluctuation of inflation rate $\dot{P}$. In barter system, there only exist phase diagrams $r_{r} \sim \dot{r}_{r}$ and $r_{r} \sim \dot{Y}_{r}$ but not $r_{r} \sim \dot{M}$ and $\dot{P} \sim \theta \ddot{P}$, the periodic change of $r_{r}$ still exists without money. Therefore, the periodic variation of $\dot{P}$ is determined by the periodic variation of $r_{r}$.

In order to eliminate the fluctuation of $\dot{P}$ or make $\dot{P} \equiv 0$ in a system in which currency are medium of exchange, the government could take two measures: firstly, to restrain the fluctuation of $r_{r}$; secondly, to control $\overline{\dot{M}}$ and therefore cause the change of $\overline{\dot{P}}$ to offset the change of $\tilde{\dot{P}}$. The first measure is usually applied by planned economy, and the second one need to make sure the change of $\overline{\dot{M}}$ would not cause the change of $\bar{r}_{r}$, otherwise there would be unexpected consequences.

As shown in Figure 14, assume $\overline{\dot{M}}$ keep unchanged, we can discuss the impacts of $\bar{r}_{r}$ on $\dot{P}$. When $\bar{r}_{r}$ drops from $\left(\bar{r}_{r}\right)_{0}$ to $\left(\bar{r}_{r}\right)_{1}$, the phase diagram 

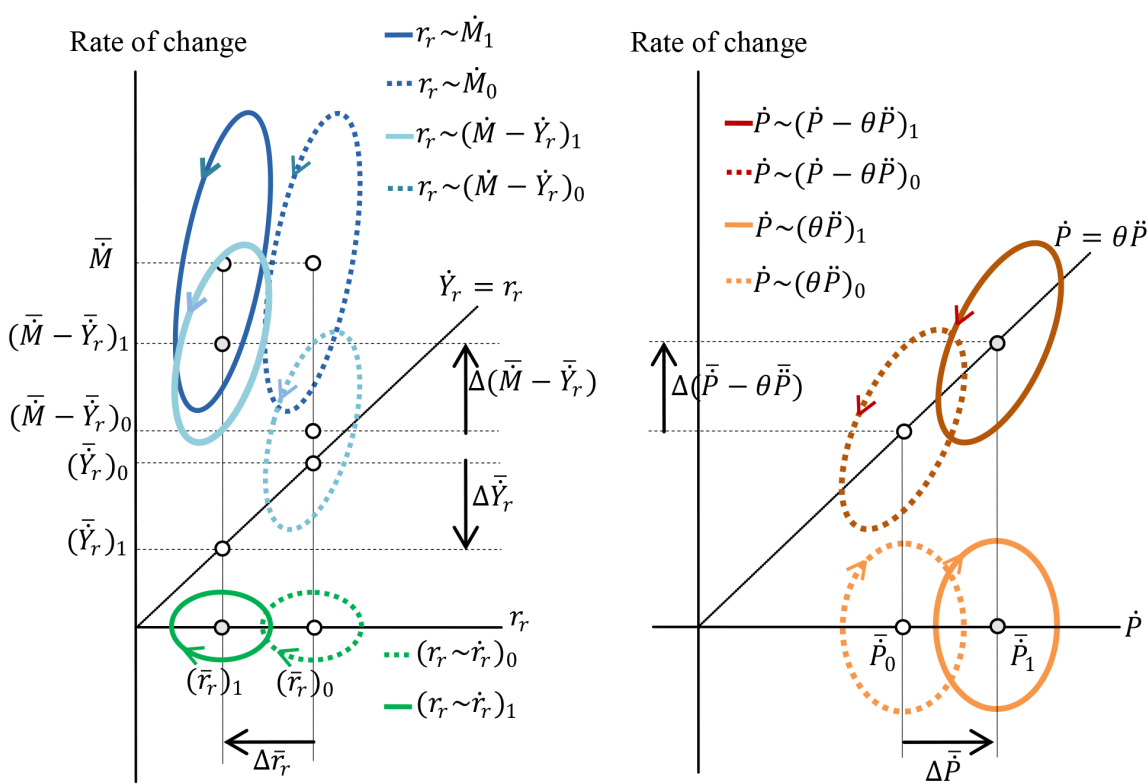

Figure 14. Impacts of changing $\bar{r}_{r}$ on $\bar{P}$, Notice: 1) This Figure is a simplified version of Figure 11, there is no phase diagrams $r_{r} \sim \dot{Y}_{r}$ and $\left.r_{r} \sim\left(-\dot{Y}_{r}\right) .2\right)$ Assume $\overline{\dot{M}}$ stay unchanged, then the smaller value of $\bar{r}_{r}$, the further place of the core variable $\overline{\dot{P}}$ towards right.

$r_{r} \sim \dot{Y}_{r}$ moves downward along the slash line, and the phase diagram $r_{r} \sim\left(\dot{M}-\dot{Y}_{r}\right)$ moves from $r_{r} \sim\left(\dot{M}-\dot{Y}_{r}\right)_{0}$ to $r_{r} \sim\left(\dot{M}-\dot{Y}_{r}\right)_{1}$. Correspondingly, the phase diagram $\dot{P} \sim(\dot{P}-\theta \ddot{P})$ and $\dot{P} \sim \theta \ddot{P}$ move upward and rightward, respectively. This means that the decline in real interest rates will cause the price level to rise. Even if the increase of $\overline{\dot{M}}$ and the increase of $\bar{r}_{r}$ occur at the same time, the change of $\overline{\dot{P}}$ and $\overline{\dot{M}}$ are in the same direction, the change of $\overline{\dot{P}}$ and $\bar{r}_{r}$ are the opposite direction, so $\overline{\dot{P}}$ would not necessarily rise.

We can analyze the change of $\dot{P}$ by the inflation equation as in Figure 13 and Figure 14, but we do not know whether $\overline{\dot{M}}$ and $\bar{r}_{r}$ affect each other, or in other words, when the increase or decrease of $\overline{\dot{M}}$ leads to the rise or drop of $\overline{\dot{P}}$, whether $\bar{r}_{r}$ change along with the change of $\overline{\dot{P}}$.

Although the inflation equation does not tell us "whether currency only has impacts on price or on both price and real output?" However, according to statistics in the United States, the change of $\overline{\dot{P}}$ usually cause the change of $\bar{r}_{r}$, especially when $\overline{\dot{P}}$ change to a significant extent. The final answer may require an analysis of the microeconomic mechanism based on the inflation equation.

\section{Conclusion}

\subsection{Hypothesis}

- The production function: $Y=A K^{\alpha} L^{\beta}$. A marginal condition: $r=\partial Y / \partial K=\alpha Y / K$.

- The quantity equation: $M=a Y$. Identity: $Y=P Y_{r}$.

- Residual: $\dot{V}=\dot{Y}-\dot{M}$, among them, $\dot{V}=\left[\dot{P} f^{\prime}(\dot{P}) / f(\dot{P})\right] \ddot{P}=\theta \ddot{P}, \quad \theta=0.1$. 


\subsection{Results}

- The Inflation equation: $\dot{P}-\theta \ddot{P}=\dot{M}-\dot{Y}_{r}$.

- The decomposition of variables: $\dot{M}=\frac{\dot{M}}{\dot{M}}, r_{r}=\bar{r}_{r}+\tilde{r}_{r}, \dot{P}=\overline{\dot{P}}+\tilde{\dot{P}}$.

\subsection{Discussion}

- By transforming of the residual $\dot{V}$, we use the analysis method of phase diagram to explain the short-term and long-term changes of the price in one equation: in the long-term, the average of $\ddot{P}$ is 0 , so $\dot{P}$ is determined by $\dot{M}$ and $\dot{Y}_{r}$; in the short-term, since $\ddot{P}$ is not $0, \dot{P}$ is determined by the phase diagram of the complex variable $\left(\dot{M}-\dot{Y}_{r}\right)$. Since these conclusions can be verified by statistical data, so they challenge the traditional theory of "demand for money". From the perspective that money is wealth, everyone wants more money than existing ones. From the perspective that money is the medium of exchange, with the popularity of non-currency payment, people realize that their demand for transactions is not a demand for trading medium (such as currency), but to transfer the real wealth of others into their own accounts through monetary policy, or to pass on financial crisis to others. Therefore, the "demand for money" analysis is difficult to explain the changes in inflation.

- Although this paper explains the factors that affect inflation, it is not able to answer whether money will affect real output at the same time as it affects prices. Omitting the fluctuation factor, if $\overline{\dot{P}}$ increases with $\overline{\dot{M}}$ increases, will $\bar{r}_{r}$ also increase. Because the change of $\overline{\dot{P}}$ is the result of the change of $\bar{r}_{r}$ and $\overline{\dot{M}}$, if the change of $\overline{\dot{M}}$ will cause the change of $\bar{r}_{r}$ by $\overline{\dot{P}}$, then $\bar{r}_{r}$ is the reason of change of $\overline{\dot{P}}$, and is affected by the change of $\overline{\dot{P}}$. This logic trap may be an important reason for the unclear role of money in the economic system. We will clarify this problem further by analyzing the Phillips curve in the next paper.

\section{References}

[1] Friedman, M. (1968) Dollars and Deficits: Living with America's Economic Problems. Prentice-Hall, Englewood Cliffs.

[2] Phillips, A.W. (1958) The Relation between Unemployment and the Rate of Change of Money Wage Rates in the United Kingdom, 1861-1957. Economica, 25, 283-299. https://doi.org/10.1111/j.1468-0335.1958.tb00003.x

[3] Diamond, P.A. (1965) National Debt in a Neoclassical Growth Model. American Economic Review, 55, 1126-1150.

[4] Baumol, W.J. (1952) The Transactions Demand for Cash: An Inventory Theoretic Approach. Quarterly Journal of Economics, 66, 545-556. https://doi.org/10.2307/1882104

[5] Tobin, J. (1965) Money and Economic Growth. Econometrica, 33, 671-684. https://doi.org/10.2307/1910352

[6] McCallum, B.T. (1983) On Non-Uniqueness in Rational Expectations Models: An Attempt at Perspective. Journal of Monetary Economics, 11, 139-168.

https://doi.org/10.1016/0304-3932(83)90028-4 
[7] Patinkin, D. (1965) Money, Interest and Prices: An Integration of Monetary and Value Theory. 2nd Edition, Row, Peterson and Co., Evanston, Harper and Row, New York.

[8] Whalen, E.L. (1966) A Reconsideration of the Precautionary Demand for Cash. Quarterly Journal of Economics, 80, 314-324. https://doi.org/10.2307/1880695

[9] Weinrobe, M.D. (1972) A Simple Model of the Precautionary Demand for Money. Southern Economic Journal, 39, 11-18. https://doi.org/10.2307/1056221

[10] Frenkel, J. and Jovanobic, B. (1980) On Transactions and Precautionary Demand for Money. Quarterly Journal of Economics, 95, 25-44. https://doi.org/10.2307/1885347

[11] Blanchard, O.J. and Fischer, S. (1989) Lectures on Macroeconomics. MIT Press, Cambridge.

[12] Buscher, H. and Frowen, S.F. (1993) The Demand for Money in Japan, the United Kingdom, West Germany and the USA: An Enpirical Study of the Evidence Since 1973. In: Frowen, S.F., Ed., Monetary Theory and Monetary Policy. New Tracks for the 1990s, Palgrave Macmillan, New York, 123-164.

https://doi.org/10.1007/978-1-349-23096-9_10

[13] Friedman, M. and Schwartz, A.J. (1956) A Monetary History of the United States, 1867-1960. Princeton University Press, Princeton.

[14] Fisher, I. (1911) The Purchasing Power of Money. 2nd Edition, Macmillan Co., New York.

[15] Keynes, J.M. (1936) The General Theory of Employment Interest and Money. Macmillan Co., Ltd., London.

[16] Friedman, M. (1956) The Quantity Theory of Money: A Restatement. In: Friedman, M., Ed., The Optimum Quantity of Money, Aldine Publishing Company, Chicago, 3-41.

[17] Zhan, M.A. and Zhan, Z. (2016) A Kind of Neither Keynesian Nor Neoclassical Model (2): The Business Cycle. Open Access Library Journal, 3, e3215. https://doi.org/10.4236/oalib.1103215

[18] Zhan, M.A. and Zhan, Z. (2016) A Kind of Neither Keynesian Nor Neoclassical Model (1): The Fundamental Equation. Open Access Library Journal, 3, e3207. https://doi.org/10.4236/oalib.1103207

Submit or recommend next manuscript to OALib Journal and we will provide best service for you:

- Publication frequency: Monthly

- 9 subject areas of science, technology and medicine

- Fair and rigorous peer-review system

- Fast publication process

- Article promotion in various social networking sites (LinkedIn, Facebook, Twitter, etc.)

- Maximum dissemination of your research work

Submit Your Paper Online: Click Here to Submit

Or Contact service@oalib.com 